\title{
Placental malaria and low birth weight in pregnant women living in a rural area of Burkina Faso following the use of three preventive treatment regimens
}

\author{
Alfred B Tiono ${ }^{1}$, Alphonse Ouedraogo ${ }^{1}$, Edith C Bougouma1, \\ Amidou Diarra ${ }^{1}$, Amadou T Konaté ${ }^{1}$, Issa Nébié ${ }^{1}$ and Sodiomon B Sirima*1,2
}

Address: ${ }^{1}$ Centre National de Recherche et de Formation sur le Paludisme, Ministère de la Santé, Ouagadougou, Burkina Faso and ${ }^{2}$ Groupe de Recherche et d'Action en Santé (GRAS), Ouagadougou, Burkina Faso

Email: Alfred B Tiono - t.alfred@fasonet.bf; Alphonse Ouedraogo - aouedraogo.cnrfp@fasonet.bf; Edith C Bougouma - eddycnrfp@yahoo.fr; Amidou Diarra - a.diarra.cnrfp@fasonet.bf; Amadou T Konaté - a.konate.cnlp@fasonet.bf; Issa Nébié - issanebie.cnlp@fasonet.bf; Sodiomon B Sirima* - s.sirima.cnlp@fasonet.bf

* Corresponding author

Published: 7 October 2009

Malaria Journal 2009, 8:224 doi:10.1/86/1475-2875-8-224
Received: 23 January 2009

Accepted: 7 October 2009

This article is available from: http://www.malariajournal.com/content/8/I/224

(c) 2009 Tiono et al; licensee BioMed Central Ltd.

This is an Open Access article distributed under the terms of the Creative Commons Attribution License (http://creativecommons.org/licenses/by/2.0), which permits unrestricted use, distribution, and reproduction in any medium, provided the original work is properly cited.

\begin{abstract}
Background: The weekly chemoprophylaxis of malaria during pregnancy with chloroquine (CQ) has become problematic with the increasing resistance of Plasmodium falciparum to this drug. There was a need to test the benefits of new strategies over the classical chemoprophylaxis. This study was conducted to provide data to the National Malarial Control Programme for an evidence-based policy change decision making process. It compares the efficacy of two IPT regimens, using chloroquine (CQ) or sulphadoxine/pyrimethamine (SP), with the classical chemoprophylaxis regimen using $\mathrm{CQ}$ in reducing the adverse outcomes of malaria infection, for the mother and the foetus.
\end{abstract}

Methods: Pregnant women attending the first antenatal care visit were randomly assigned to one of the three treatment regimens. They were subsequently followed up till delivery. Maternal, placental and cord blood samples were obtained upon delivery to check for $P$. falciparum infection.

Results: A total of 648 pregnant women were enrolled in the study. Delivery outcome were available for 423 of them. Peripheral maternal P. falciparum infection at delivery was found in $25.8 \%$ of the women. The proportion of women with maternal infection was significantly lower in the IPTP/SP group than in the CQ group $(\mathrm{P}<<0.000)$. The prevalence of placental malaria was $18.8 \%$ in the CWC/CQ group; $15.9 \%$ in the IPTP/CQ group and $10.6 \%$ in the IPTP/SP group. The incidence of LBW (weigth $<2,500 \mathrm{~g}$ ) was significantly higher among infants of mothers in the CWC/ CQ group (23.9\%) as compared with those of mothers in the IPTP/CQ (15.6\%) and IPTP/SP (II.6\%) groups $(p=0.02)$

Conclusion: Intermittent preventive treatment with SP has shown clear superiority in reducing adverse outcomes at delivery, as compared with intermittent preventive treatment with $\mathrm{CQ}$ and classical chemoprophylaxis with CQ. 


\section{Background}

In malaria endemic countries, pregnant women, along with children under five years, represent the most vulnerable group to Plasmodium falciparum infection [1,2]. Such infection often increases the risk of morbidity and mortality for the mother and her child. Indeed, in malaria stable transmission conditions, it has been shown that pregnancy associated with malaria increases the risk of maternal anaemia, stillbirths and low birth weight (LBW) $[3,4]$. Over $26 \%$ of anaemia in pregnancy is attributable to malaria, and malaria-related maternal deaths are reaching an unacceptable rate of $23 \%$ [5]. These adverse outcomes have been described as the consequences of placental sequestration of $P$. falciparum. In addition, because of this sequestration, peripheral blood film microscopy usually underestimates the prevalence of placental malaria [6,7].

In areas of high or moderate transmission, most malaria infections in pregnant women are asymptomatic and therefore will not be presented to any clinic for diagnosis and management. It is important that in such conditions, strategies to protect mothers during their pregnancy include the use of insecticide-treated material (ITM) as well as effective anti-malarial drugs used in intermittent preventive treatment (IPT) regimens and case management [8-10].

In Burkina Faso, until recently, chloroquine (CQ) was the only drug recommended by the National Malaria Control Programme for use in chemoprophylaxis against malaria in pregnant women. However, with the increasing resistance of $P$. falciparum to chloroquine and the evidence of the efficacy of IPT in pregnant women using sulphadoxine-pyrimethamine (SP), new guidelines were issued in February 2005 for a policy change regarding the prevention of malaria during pregnancy.

The aim of the present study was to provide data to the National Malarial Control Programme for an evidencebased policy change decision making process. The study has assessed the efficacy of three possible approaches being considered at the time of the study initiation. It compares the efficacy of two IPT regimens (using CQ or $\mathrm{SP}$ ) with the classical chemoprophylaxis regimen using $\mathrm{CQ}$ in reducing the adverse outcomes of malaria infection for the mother and the foetus.

\section{Methods}

\section{Study site and population}

Details on the study site and methods were published by Ouedraogo et al [11]. Briefly, the study was conducted in the Antenatal Care Unit of the Health District of Bousse, a district located around $50 \mathrm{~km}$ north-west of Ouagadougou, the capital of Burkina Faso. The district covers a total population of 124,285 inhabitants. The majority of this population is farmers, illiterate, and belongs to the Mossi ethnic group.

The health district is situated within the Mossi Central Plateau, in the Sudano-Sahelian zone, with a tropical climate characterized by two seasons: the dry season (November May), in which malaria transmission is very low, and the rainy season (June - October), in which malaria transmission is high. The annual rainfall is in the range of 600 to $900 \mathrm{~mm}$. The main malaria vectors are Anopheles gambiae, Anopheles arabiensis and Anopheles funestus. Two chromosomal forms each of An. gambiae and An. funestus are sympatric in the study area. The annual entomological inoculation rates range from 10 to 500 infective bites per individual. Plasmodium falciparum is responsible for more than $90 \%$ of malaria infections.

In terms of infrastructure, the Health District includes the District Hospital, which serves as referral center for 11 community health clinics named Centre de Santé et de Promotion Sociale (CSPS). The district hospital comprises a medical unit, a surgery unit and the Child and Maternal Health Unit (CMHU). Antenatal care and family planning services are offered by the CMHU. Therefore, the study staff recruiting team was based there to screen and enrol women seeking antenatal care who fulfilled the study inclusion criteria. Among other criteria, eligible women were required to be permanent residents of the Health District area with a gestational age between 15 and 36 weeks at the time of the visit, and to have provided freely given written informed consent. All women who were otherwise eligible but who had a history of allergic reaction to any of the study drugs (CQ and SP) were excluded.

\section{Selection, randomization and treatment of the study participants}

In Burkina, three antenatal care unit visits are recommended during pregnancy. The initial visit $\left(\mathrm{ANC}_{1}\right)$ is scheduled between the $15^{\text {th }}$ and $25^{\text {th }}$ week of gestational age; the second visit $\left(\mathrm{ANC}_{2}\right)$ is scheduled between the $28^{\text {th }}$ and $32^{\text {nd }}$ week of gestational age, and the third visit $\left(\mathrm{ANC}_{3}\right)$ is scheduled between the $33^{\text {rd }}$ and the $36^{\text {th }}$ week of gestational age.

Pregnant women undergoing their $\mathrm{ANC}_{1}$ who were willing to participate in the study were assessed with regard to the inclusion and exclusion criteria. Eligible women were randomly assigned to one of the three treatment arms using a computer generated random list.

The study pharmacist, who did not take part in any other activities in the study, prepared an individual randomization code envelope from the randomization list. The sealed envelopes were sequentially numbered. When an eligible woman joined the study, the envelope with the 
lowest available number was opened, revealing the assignment of the participant to the appropriate treatment arm.

The first treatment arm was classical weekly chemoprophylaxis using chloroquine (CWC/CQ). Participants in this arm received, upon enrolment, a curative dose of CQ (600 $\mathrm{mg} /$ day at Day 0, then $300 \mathrm{mg} /$ day at Day 1 and Day 2) followed by $300 \mathrm{mg} /$ week until 6 weeks post delivery. The second and third treatment arms consisted of intermittent preventive treatment in pregnancy (IPTp) regimens. For the second arm (IPTp/CQ), the IPTp regimen was a complete cure with $\mathrm{CQ}$ at each of the antenatal care visits $\left(\mathrm{ANC}_{1}, \mathrm{ANC}_{2}\right.$ and $\left.\mathrm{ANC}_{3}\right)$. A total dose of $600 \mathrm{mg} /$ day of CQ was administered at Day 0, then $300 \mathrm{mg} /$ day at Day 1 and Day 2. In the third arm (IPTp/SP), the IPTp regimen consisted of a single curative dose of $1.5 \mathrm{~g} / 0.075 \mathrm{~g}$ sulphadoxine pyrimethamine at each of the antenatal care visits $\left(\mathrm{ANC}_{1}, \mathrm{ANC}_{2}\right.$ and $\left.\mathrm{ANC}_{3}\right)$. The treatment administration was not supervised. Each woman was given medication, according to her treatment arm, for selfadministration after leaving the clinic.

\section{Follow-up of the study participants}

Enrolled pregnant women were followed up to delivery. At enrolment and subsequent visits, the study midwife collected the study data using a standardized questionnaire. During the pregnancy, the following data were collected: sociodemographic factors (age, village, school attendance, marital status), obstetrical past history (gravidity, parity), pregnancy related factors (number of ANC visits, oedema, malaria episode), and malaria prevention measures (chemoprophylaxis and use of bed nets). A physical examination was performed to measure axillary temperature, blood pressure, weight and uterine height.

A blood sample was obtained by fingerprick for a blood smear and haemoglobin $(\mathrm{Hb})$ was measured using a portable hemocue (HemoCue ${ }^{\circledast B} \mathrm{AB}$, Ängelholm, Sweden). Anaemia was defined as $\mathrm{Hb}<11 \mathrm{~g} / \mathrm{dL}$. An $\mathrm{Hb}$ level between 8 and $11 \mathrm{~g} / \mathrm{dL}$ was considered mild to moderate anemia and an $\mathrm{Hb}$ level $\leq 8 \mathrm{~g} / \mathrm{dL}$ as severe anaemia.

In participants diagnosed with fever (axillary $\mathrm{T}^{\circ} \geq$ $37.5^{\circ} \mathrm{C}$ ), a malaria rapid diagnosis test $\left(\mathrm{OPTimal}^{\circledR}\right.$ ) was done to allow prompt management of the case. Results of the impact of the three treatment arms in terms of maternal infection and prevalence of maternal anaemia have been published elsewhere by Ouedraogo et al [11]

At delivery, data regarding newborn characteristics (vital status at birth, birth weight, sex and the presence of twins or malformation) were collected. Birth weight was measured using an electronic digital scale ( \pm 10 grams) (Tanita Corporation, Tokyo, Japan). In addition, cord blood sam- ples were obtained for smears and placental blood smears were obtained from the maternal side of the placenta. A malaria smear was also obtained from the mother by fingerprick. No sample was obtained from the neonate to check for malaria parasites.

\section{Management of anaemia and malaria cases}

Study participants found to be anaemic at ANC visits ( $\mathrm{Hb}$ level $<11 \mathrm{~g} / \mathrm{dL}$ ) were treated, based on national guidelines, with ferrous sulfate $(200 \mathrm{mg})$ and folic acid $(0.25$ $\mathrm{mg}$ ) given as a single combined tablet daily for 30 days. Confirmed cases of malaria (axillary $\mathrm{T}^{\circ} \geq 37.5^{\circ} \mathrm{C}$ and with a positive rapid diagnosis test) were given treatment with quinine, based on the national malaria treatment guidelines for pregnant women.

\section{Laboratory methods}

All blood films (maternal, placental and cord) were stained with $10 \%$ Giemsa for $45 \mathrm{~min}$, and read at the Centre National de Recherche et de Formation sur le Paludisme (CNRFP) immunoparasitology laboratory in Ouagadougou. For thick films, parasites and leukocytes were counted in the same fields until 500 leukocytes were counted. Parasite densities were estimated using an assumed leukocyte count of 8,000 leukocytes $/ \mu \mathrm{L}$. Thin films were then used to determine species when thick films were positive. All slides were double read by two independent microscopists. If the ratio of densities from the first two readings were $>1.5$ or $<0.67$ or if $<30$ parasites were counted with a difference in the number of parasites $>10$, the slide was evaluated a third time. The final result was the geometric mean of the parasite density of the two most concordant results of the three readings. When the discordance was only in terms of positivity, the slide was also evaluated a third time and the definitive result was based on the majority verdict for positivity. Placental histology was not performed.

\section{Statistical analysis}

The data collected in the study questionnaire were verified then double entered using Epi-info 6.04 fr (CDC, Atlanta, USA). Data were analysed using Epi-info and Sata 7.0. Analysis was by intent-to-treat. The analysis included data from births of all enrolled participants who had taken the study treatment at least once and who delivered at the study centre clinic for which data are available. Continuous normally distributed data were described by the mean and standard deviation, and non-normally distributed data by the median or geometrical mean and range.

Proportions were compared using the Chi square test and normally distributed continuous variables were compared using Student's $t$ test. Statistical tests were considered significant when the two-sided $\mathrm{P}$ value was $<0.05$. 


\section{Ethical considerations}

The study was initially discussed with health authorities and local leaders to obtain their assent. The study was submitted to and approved by the National Ethical Review Committee in Burkina Faso and the Ethical Committee of WHO/TDR. A written informed consent was obtained from all the women prior to their enrolment in the study. For illiterate mothers, the informed consent discussion process was witnessed by an impartial individual.

\section{Results}

\section{Characteristics of the study participants at enrolment}

The trial profile is provided in Figure 1. In total, 648 pregnant women were recruited and randomized to one of the three study arms. Baseline characteristics were similar within the study groups (Table 1). The mean age (SD) of the participants was 23.7 (5.9) and 39.7\% of them (257) were primigravidae. The primigravidae and secundigravidae represent $58.7 \%$ of the total sample size. Over half of the women (50.8\%) were parasitaemic at enrolment; this proportion was significantly higher for women randomized to the IPTp/SP group ( $\mathrm{p}=0.005)$. Overall, the geometric mean of the parasite density was 855.4 (95\% ci 691.6-1058.06). There was no difference between treatment groups. The rate of use of insecticide impregnated materials (bed nets and/or curtains) was very low in the study population and was comparable across the study groups.

One woman $(0.24 \%)$ in the IPTp/SP group died during the delivery as the consequence of eclampsia. Other complications during the delivery included peripartum haemorrhage $(1.18 \%)$, placental retention $(1.9 \%)$, and retroplacental haemorrhage $(1.4 \%)$. Two stillbirths $(0.4 \%)$ were recorded.

\section{Placental malaria and low birth weight}

A total of 423 women were followed up until delivery; they were similarly distributed in the three treatment groups (see Table 2). Overall, peripheral maternal P. falciparum infection at delivery was found in $25.8 \%$ of the women. The proportion of women with maternal infection was significantly lower in the IPTp/SP group than in the CQ group $(\mathrm{P}<<0.000)$. Among all the delivering primigravidae, the proportion of women with maternal infection was almost double in the CWC/CQ group compared with the IPT groups. The difference was statistically significant ( $\mathrm{p}<0.000$ ). Among the secundigravidae, although the highest proportion of infected mothers was observed in the IPT groups (47.8\% for the IPTp/CQ group and $22.7 \%$ for the IPTp/SP group) as compared to the CWC/CQ group (20.0\%), this result did not reach significance level $(\mathrm{p}=0.07)$.

The prevalence of placental malaria was $18.8 \%$ in the CWC/CQ group; $15.9 \%$ in the IPTp/CQ group and $10.6 \%$ in the IPTp/SP group. These trends remained unchanged after stratification according to level of gravidity (Table 2). Statistically significant differences were only found between the CWC/CQ group and the IPTp/SP group ( $\mathrm{p}=$ $0.049)$.

After adjustment, women in the IPTp/SP group had a lower risk of placental malaria when compared with those in the CWC/CQ group ( $\mathrm{OR}=0.44$ (95\% ci 0.22-0.90), p $=0.025)$. No significant difference was observed between the women in the IPTp/CQ group and those in the CWC/ $\mathrm{CQ}$ group $(\mathrm{OR}=0.75(95 \%$ ci $0.4-1.41), \mathrm{p}=0.39)$. The mean birth weight was $2,802 \mathrm{~g}(1,100$ to 4,100$)$; there were no differences among the treatment groups in mean birth weight.

In total, $17.3 \%$ of the newborns had a low birth weight (LBW) (weight $<2,500 \mathrm{~g}$ ). The incidence of LBW was significantly higher among infants of mothers in the CWC/ CQ group (23.9\%) as compared with those of mothers in the IPTp/CQ (15.6\%) and IPTp/SP (11.6\%) groups ( $\mathrm{p}=$ $0.02)$. The use of IPTp either with CQ or SP was associated with lower risk of LBW; thus, the adjusted risk of LBW was significantly lower for infants of mothers in the IPTp/SP group as compared with those from the CWC/CQ group $(\mathrm{OR}=0.38$ (95\% ci (0.19-0.72), $\mathrm{p}=0.004)$. A similar situation was observed when comparing infants of mothers in the IPTp/CQ and those of mothers in the CWC/CQ group $(\mathrm{OR}=0.53,95 \% \mathrm{ci}(0.29-0.98), \mathrm{p}=0.045)$.

As presented in Table 2, among primigravidae, the proportion of infants with LBW was significantly higher in the CWC/CQ group (40.9\%, versus $18.9 \%$ in IPTp/CQ and $14.5 \%$ in IPTp/SP groups, $\mathrm{p}=0.004)$. In secundigravidae and multigravidae mothers, the proportion of infants with LBW was also higher in the CWC/CQ group, but this difference did not reach statistical significance.

Cord blood parasitaemia was found in six cases; four of them were from the CWC/CQ group, one in the IPTp/CQ group and one in the IPTp/SP group. The difference was not statistically significant $(\mathrm{p}=0.19)$.

\section{Discussion}

This study compared the efficacy of three treatment regimens for the prevention of placental malaria: classical chemoprophylaxis using $\mathrm{CQ}$, intermittent preventive treatment using $\mathrm{CQ}$, and intermittent preventive treatment using two doses of SP.

This study was conducted at the time of a transition period in Burkina Faso; CWC/CQ was still the regimen recommended by the National Malaria Control Programme for preventing malaria during pregnancy, but the declining efficacy of CQ had lead to the initiation of discussion of a change in this policy. 


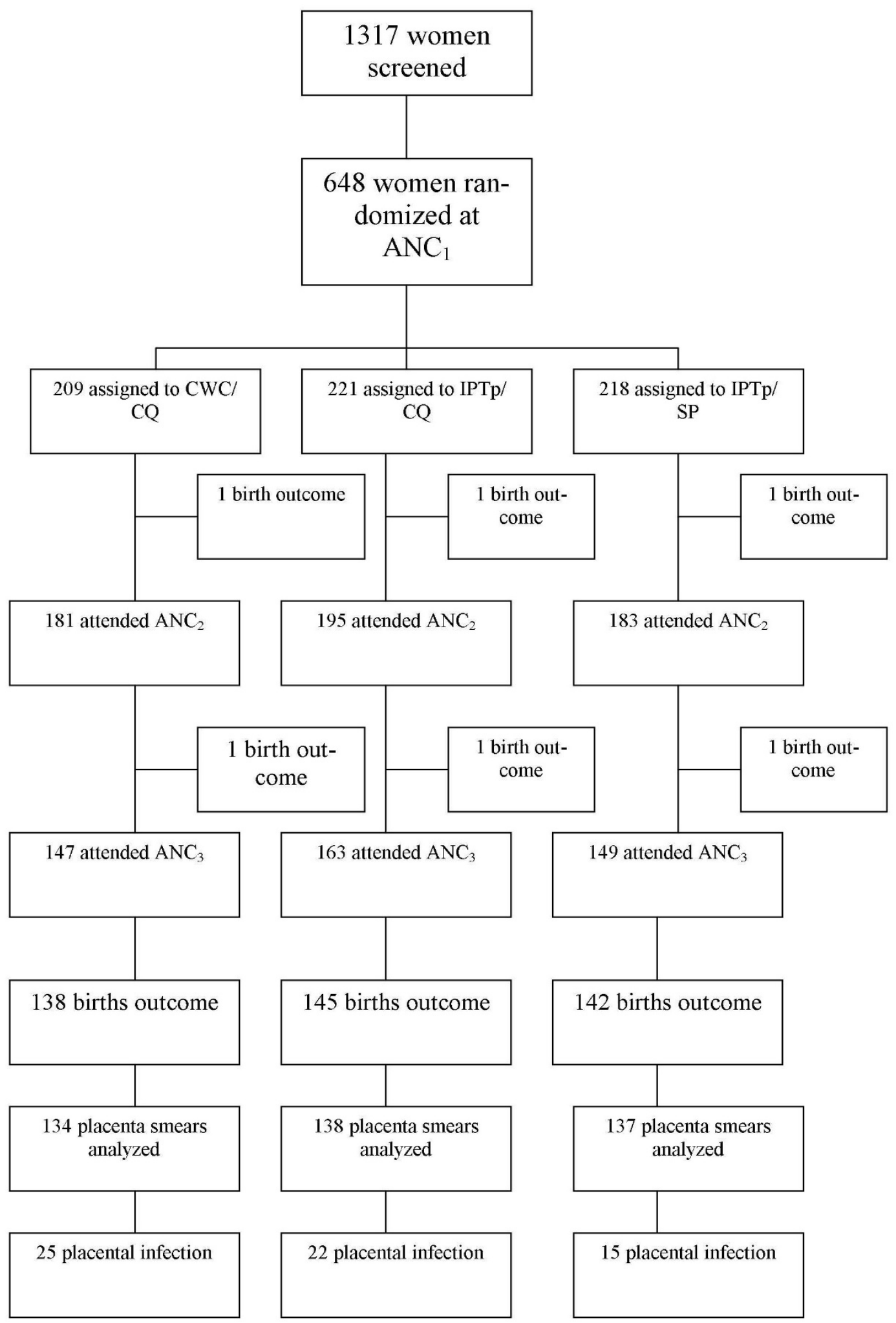

Figure I

The trial profile. 
Table I: Baseline characteristics of the study participants

\begin{tabular}{|c|c|c|c|c|c|}
\hline Characteristics & $\begin{array}{c}\text { CWC/CQ } \\
n=209\end{array}$ & $\begin{array}{l}\text { IPTp/CQ } \\
N=22 I\end{array}$ & $\begin{array}{l}\text { IPTp/SP } \\
n=218\end{array}$ & All participants $N=648$ & $\mathbf{P}$ \\
\hline $\begin{array}{l}\text { Age } \\
\text { (mean } \pm \text { SD; range) }\end{array}$ & $24.3 \pm 6.3(16 ; 51)$ & $23.3 \pm 5.7(17 ; 42)$ & $23.6 \pm 5.8(17 ; 45)$ & $23.7 \pm 5.9(16 ; 51)$ & 0.16 \\
\hline $\begin{array}{l}\text { Parity } \\
\text { (mean } \pm \mathrm{SD} \text {; range) }\end{array}$ & $1.7 \pm 0.01(0 ; 8)$ & $\begin{array}{l}\mathrm{I} .5 \pm 0.0 \mathrm{I} \\
(0 ; 9)\end{array}$ & $\begin{array}{l}1.4 \pm 0.0 \mathrm{I} \\
(0 ; 7)\end{array}$ & $\begin{array}{l}1.5 \pm 0.01 \\
(0 ; 9)\end{array}$ & 0.07 \\
\hline Primigravidae (\%) & 32.2 & 42.5 & 44.0 & 39.7 & 0.02 \\
\hline Secundigravidae (\%) & 21.2 & 19.0 & 17.0 & 19.0 & 0.56 \\
\hline Multigravidae (\%) & 46.6 & 38.5 & 39.0 & 41.3 & 0.18 \\
\hline $\begin{array}{l}\text { Weight } \\
\text { (mean } \pm \text { SD; range) }\end{array}$ & $54.6 \pm 6.3(4 I, 80)$ & $54.8 \pm 8.3(39 ; 79)$ & $55.7 \pm 8.2(40 ; 105)$ & $55.2 \pm 7.7(39 ; 105)$ & 0.54 \\
\hline $\begin{array}{l}\text { Gestational age } \\
(\text { mean } \pm \text { SD) }\end{array}$ & $21.8 \pm 2.8$ & $21.6 \pm 2.8$ & $21.75 \pm 2.7$ & $21.75 \pm 2.7$ & 0.67 \\
\hline $\begin{array}{l}\text { Uterine fundal Height } \\
\text { (mean } \pm \text { SD; range) }\end{array}$ & $21.4 \pm 3.3(13 ; 30)$ & $21.2 \pm 3.2(13 ; 30)$ & $\begin{array}{l}21.2 \pm 2.8 \\
(14 ; 27)\end{array}$ & $21.3 \pm 3.1(13 ; 30)$ & 0.66 \\
\hline Parasitaemia (mean, $95 \% \mathrm{Cl}$ ) & $666.6(450.9985 .6$ & $\begin{array}{c}908.1 \\
(636.5-1295.8)\end{array}$ & $\begin{array}{c}951.8 \\
(661.5-1369.4)\end{array}$ & $855.4(691.6-1058.06)$ & 0.27 \\
\hline Parasitaemia (\%) & $41.5 \%$ & $55.0 \%$ & $55.3 \%$ & $50.8 \%$ & 0.005 \\
\hline $\begin{array}{l}\text { Haemoglobin level } \\
\text { (mean } \pm \text { DS; range) }\end{array}$ & $10.9 \pm 1.5(5.1 ; 15.2)$ & $10.9 \pm 1.4(7.0 ; 14.0)$ & $\begin{array}{l}10.4 \pm 1.3 \\
(7.8 ; 14.6)\end{array}$ & $11.0 \pm 1.4(5.1 ; 15.2)$ & 0.81 \\
\hline Impregnated bed nets (\%) & 4.3 & 6.8 & 5.0 & 5.4 & 0.50 \\
\hline Non-impregnated bed nets (\%) & 9.6 & 6.3 & 6.9 & 7.56 & 0.39 \\
\hline Impregnated curtains (\%) & 2.9 & 2.3 & 2.3 & 2.5 & 0.89 \\
\hline Non-impregnated curtains (\%) & 56.3 & 67.0 & 59.6 & 61.0 & 0.06 \\
\hline
\end{tabular}

The IPTp/SP regimen was more effective than the other two regimens in preventing placental malaria. No difference was found between the IPTp/CQ and the CWC/CQ regimens. Significant differences were only found between the CWC/CQ and IPTp/SP groups, suggesting that, in addition to the high resistance of $P$. falciparum to CQ in the area, $[12,13]$ the low efficacy observed with the $\mathrm{CWC} / \mathrm{CQ}$ is probably also related to the issue of compliance. Compliance issues with this type of treatment regimen have been commonly observed in women living in settings comparable to those of our study population.

The risk of placental malaria was significantly higher in the CWC/CQ as compared to the IPTp/SP group. This finding is consistent with those reported in similar studies which have shown that IPTp with SP is more efficacious than chemoprophylaxis with CQ in preventing placental malaria [14-16].

The IPTp/SP regimen was also more efficacious in reducing the proportion of LBW as compared with the two other CQ regimens. These findings are consistent with what has been reported in similar studies [14,17]. The efficacy of all three-treatment regimens in preventing maternal infection, placental malaria, and low birth weight increased with gravidity. This finding confirms the high burden of malaria for women with low gravidity in areas with intense parasite transmission. Indeed, in high malaria endemicity settings, primigravidae and, to a lesser extent, secundigravidae are known to be more highly affected than other parities [18].

The proportion of premature delivery was unexpectedly high in all three-treatment regimens. No significant differences were observed between the groups. Considering the efficacy of IPTp/SP in preventing placental malaria infection, and CQ's potential antipyretic effect (mediated by inhibition of tumour necrosis factor) [19], one may expect lower premature delivery rates comparable with those reported in other studies using the same anti-malarial drugs $[14,15,20]$. It is possible that in this case, the gestational ages were overestimated in some cases due to a recall bias (of the date of last menstrual period, which is used to estimate the gestational age at the first antenatal clinic visit) by the mothers.

\section{Conclusion}

From the perspective of reducing the adverse consequences of malaria on pregnancy outcomes, IPTp/SP has shown clear superiority as compared with IPTp/CQ and classical chemoprophylaxis with $\mathrm{CQ}$. These results indicate that IPTp/SP treatment could be adopted as the official recommended treatment for pregnant women, while alternative drugs for IPT are being investigated.

\section{Competing interests}

The authors declare that they have no competing interests. 
Table 2: Outcomes at delivery

\begin{tabular}{|c|c|c|c|c|c|}
\hline Characteristics & $\begin{array}{l}\text { CWC/CQ } \\
(n=137)\end{array}$ & $\begin{array}{l}\text { IPTp/CQ } \\
(n=145)\end{array}$ & $\begin{array}{l}\text { IPTp/SP } \\
(n=14 I)\end{array}$ & $\begin{array}{c}\text { All } \\
(N=423)\end{array}$ & $\mathbf{P}$ \\
\hline \multicolumn{6}{|l|}{ Maternal parasitaemia } \\
\hline Overall & 32.8 & 30.9 & 13.9 & 25.8 & 0.00 \\
\hline Primigravidae & 57.8 & 36.4 & 16.9 & 35.2 & 0.00 \\
\hline Secundigravidae & 20.0 & 47.8 & 22.7 & 30.0 & 0.07 \\
\hline Multigravidae & 19.7 & 19.7 & 7.1 & 15.7 & 0.10 \\
\hline \multicolumn{6}{|l|}{ Placental malaria (\%) } \\
\hline Overall & 18.8 & 15.9 & 10.6 & 15.6 & 0.14 \\
\hline Primigravidae & 31.1 & 25.4 & 15.9 & 23.4 & 0.16 \\
\hline Secundigravidae & 17.9 & 20.0 & 13.6 & 17.3 & 0.84 \\
\hline Multigravidae & 10.8 & 4.9 & 3.5 & 6.6 & 0.22 \\
\hline Placental malaria Parasite densities (mean* $95 \% \mathrm{Cl}$ ) & $\begin{array}{c}666.7 \\
(244.8-1815.75)\end{array}$ & $\begin{array}{c}1535.8 \\
(560.8-4205.9)\end{array}$ & $1030.1(260.8-4069.0)$ & $\begin{array}{c}996.5 \\
(544.9-1822.3)\end{array}$ & 0.53 \\
\hline \multicolumn{6}{|l|}{ Cord blood parasitaemia (\%) } \\
\hline Overall & 2.9 & 0.7 & 0.7 & 1.4 & 0.19 \\
\hline Primigravidae & 2.2 & 1.7 & 1.6 & 1.8 & 0.97 \\
\hline Secundigravidae & 7.14 & 0 & 0 & 2.7 & 0.18 \\
\hline Multigravidae & 1.54 & 0 & 0 & 0.6 & 0.40 \\
\hline Birth weight (mean, range) & $\begin{array}{c}2749 \\
(1100-3900)\end{array}$ & $\begin{array}{c}2819 \\
(1500-4100)\end{array}$ & $\begin{array}{c}2836 \\
(1500-4000)\end{array}$ & $\begin{array}{c}2802 \\
(1100-4100)\end{array}$ & 0.15 \\
\hline \multicolumn{6}{|l|}{ Low birth weight** (\%) } \\
\hline Overall & 23.9 & 15.6 & 11.4 & 16.9 & 0.02 \\
\hline Primigravidae & 40.9 & 18.9 & 14.5 & 23.2 & 0.004 \\
\hline Secundigravidae & 18.5 & 16.00 & 14.3 & 16.4 & 0.92 \\
\hline Multigravidae & 16.9 & 13.6 & 8.9 & 13.3 & 0.43 \\
\hline Premature delivery & $28.3 \%$ & $26.2 \%$ & $30.3 \%$ & $28.2 \%$ & 0.75 \\
\hline Spontaneous abortion & $1.5 \%$ & $1.4 \%$ & $0.7 \%$ & $1.2 \%$ & 0.81 \\
\hline
\end{tabular}

* Geometric mean

** $\mathrm{CWC} / \mathrm{CQ}=134 ; \mathrm{IPT} / \mathrm{CQ}=138 ; \mathrm{IPT} / \mathrm{SP}=137$

\section{Authors' contributions}

SBS conceived the study and its design; he coordinated the data collection, the analysis and interpretation of the results and the review of the manuscript. ABT participated to the design of the study, the data collection and analysis and has drafted the manuscript. AO, ECB, AD, ATK and IN participated in the data collection and data analysis and interpretation. All authors have read and approved the final manuscript.

\section{Acknowledgements}

We acknowledge the valuable contribution of the women who participated in this study. A special thanks to all the staff of the health District of Boussé, Mlle Badolo Claire and Mlle Bationo for their help during the investigations. We also thank the Lab staff of the Centre National de Recherche et de Formation sur le Paludisme. This investigation received financial support from the UNDP/World Bank/WHO Special Programme for Research and Training in Tropical Diseases (TDR) (Project ID number: A20789)

\section{References}

I. Brabin BJ: An analysis of malaria in pregnancy in Africa. Bull World Health Organ 1983, 61:1005-1016.

2. Nosten F, ter Kuile F, Maelankirri L, Decludt B, White NJ: Malaria during pregnancy in an area of unstable endemicity. Trans $R$ Soc Trop Med Hyg 1991, 85:424-429.

3. Menendez C, Ordi J, Ismail MR, Ventura PJ, Aponte JJ, Kahigwa E, Font $\mathrm{F}$, Alonso PL: The impact of placental malaria on gestational age and birth weight. J Infect Dis 2000, 181:1740-1745.
4. Ordi J, Ismail MR, Ventura PJ, Kahigwa E, Hirt R, Cardesa A, Alonso $\mathrm{PL}$, Menendez C: Massive chronic intervillositis of the placenta associated with malaria infection. Am J Surg Pathol 1998, 22:1006-1011.

5. Guyatt HL, Snow RW: The epidemiology and burden of Plasmodium falciparum-related anemia among pregnant women in sub-Saharan Africa. Am J Trop Med Hyg 200I, 64:36-44.

6. Mankhambo L, Kanjala M, Rudman S, Lema VM, Rogerson S): Evaluation of the OptiMAL rapid antigen test and species-specific PCR to detect placental Plasmodium falciparum infection at delivery. J Clin Microbiol 2002, 40(1):155-158.

7. Mockenhaupt FP, Ulmen U, von Gaertner C, Bedu-Addo G, Bienzle U: Diagnosis of placental malaria. I Clin Microbiol 2002, 40:306-308.

8. Gamble C, Ekwaru PJ, Garner P, ter Kuile FO: Insecticide-treated nets for the prevention of malaria in pregnancy: a systematic review of randomised controlled trials. PLoS Med 2007, 4:e I07.

9. Nahlen BL: Rolling back malaria in pregnancy. N Engl J Med 2000, 343:65I-652.

10. WHO: A strategic Framework for Malaria Prevention and Control during Pregnancy in the Africa Region. World Health Organization Regional Office for Africa 2004.

II. Ouedraogo A, Bougouma EC, Diarra A, Konate AT, Nebie I, Tiono $A B$, Sirima $S B$ : [Comparative impact of three malaria preventive regimens during pregnancy on maternal anemia due to malaria in Burkina Faso]. Med Mal Infect 2008, 38: I80-186.

12. Coulibaly SO, Nezien D, Traore S, Kone B, Magnussen P: Therapeutic efficacy of sulphadoxine-pyrimethamine and chloroquine for the treatment of uncomplicated malaria in pregnancy in Burkina Faso. Malar J 2006, 5:49.

13. Sirima SB, Sawadogo R, Moran AC, Konate A, Diarra A, Yameogo M, Parise ME, Newman RD: Failure of a chloroquine chemoprophylaxis program to adequately prevent malaria during preg- 
nancy in Koupela District, Burkina Faso. Clin Infect Dis 2003, 36:1374-1382.

14. Kayentao K, Kodio M, Newman RD, Maiga H, Doumtabe D, Ongoiba A, Coulibaly D, Keita AS, Maiga B, Mungai M Parise ME, Doumbo O: Comparison of intermittent preventive treatment with chemoprophylaxis for the prevention of malaria during pregnancy in Mali. J Infect Dis 2005, 191:109-1 16.

15. Parise ME, Ayisi JG, Nahlen BL, Schultz LJ, Roberts JM, Misore A, Muga R, Oloo AJ, Steketee RW: Efficacy of sulfadoxinepyrimethamine for prevention of placental malaria in an area of Kenya with a high prevalence of malaria and human immunodeficiency virus infection. Am J Trop Med Hyg 1998, 59:813-822.

16. Schultz LJ, Steketee RW, Macheso A, Kazembe P, Chitsulo L, Wirima $\mathrm{JJ}$ : The efficacy of antimalarial regimens containing sulfadoxine-pyrimethamine and/or chloroquine in preventing peripheral and placental Plasmodium falciparum infection among pregnant women in Malawi. Am J Trop Med Hyg 1994, 5 I:515-522.

17. Sirima SB, Cotte AH, Konate A, Moran AC, Asamoa K, Bougouma EC, Diarra A, Ouedraogo A, Parise ME, Newman RD: Malaria prevention during pregnancy: assessing the disease burden one year after implementing a program of intermittent preventive treatment in Koupela District, Burkina Faso. Am J Trop Med Hyg 2006, 75:205-2II.

18. McGregor IA, Wilson ME, Billewicz WZ: Malaria infection of the placenta in The Gambia, West Africa; its incidence and relationship to stillbirth, birthweight and placental weight. Trans $R$ Soc Trop Med Hyg 1983, 77:232-244.

19. Kwiatkowski D, Bate C: Inhibition of tumour necrosis factor (TNF) production by antimalarial drugs used in cerebral malaria. Trans R Soc Trop Med Hyg 1995, 89:215-216.

20. Denoeud L, Fievet N, Aubouy A, Ayemonna P, Kiniffo R, Massougbodji $A$, Cot M: Is chloroquine chemoprophylaxis still effective to prevent low birth weight? Results of a study in Benin. Malar J 2007, 6:27.

Publish with Bio Med Central and every scientist can read your work free of charge

"BioMed Central will be the most significant development for disseminating the results of biomedical research in our lifetime. "

Sir Paul Nurse, Cancer Research UK

Your research papers will be:

- available free of charge to the entire biomedical community

- peer reviewed and published immediately upon acceptance

- cited in PubMed and archived on PubMed Central

- yours - you keep the copyright

Submit your manuscript here:

http://www.biomedcentral.com/info/publishing_adv.asp
BioMedcentral 